\section{circASXL1-1 regulates BAP1 deubiquitinase activity}

\section{in leukemia}

Mutations in epigenetics remodelers such as ASXL1 (additional sex combs 1) have been identified in acute myeloid leukemia, chronic myelomonocytic leukemia and myelodysplastic syndrome and are associated with poor overall survival. ${ }^{1}$ Some ASXL1 mutations resulting in a loss of protein expression have been shown to contribute to myeloid transformation through loss of H3K27me3 expression at the HOX gene loci. ${ }^{2}$ On the other hand, some ASXL1 mutations result in the expression of a truncated protein, which can bind and activate BRCA-1 associated protein 1 (BAP1), a deubiquitinase enzyme, to form the polycomb repressive deubiquitinase complex (PR-DUB). A hyperactive PR-DUB de-represses target genes by deubiquitinating H2AK119 leading to impaired hematopoietic stem cell differentiation and leukemogenesis. ${ }^{3-5}$ Conversely, reduced BAP1 activity is sufficient to halt the leukemogenesis driven by truncated ASXL1 protein, ${ }^{6}$ highlighting the importance of a balanced ASXL1-BAP1 axis in normal hematopoiesis.
A

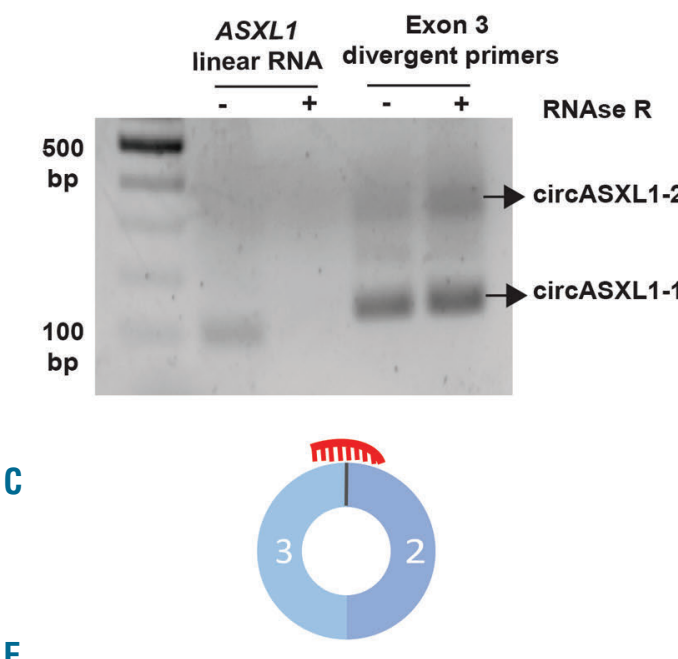

E

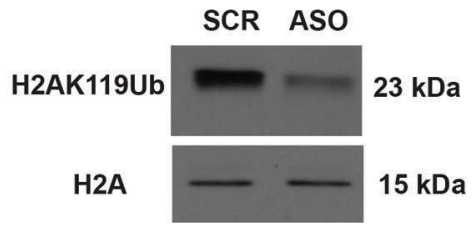

$\mathbf{F}$

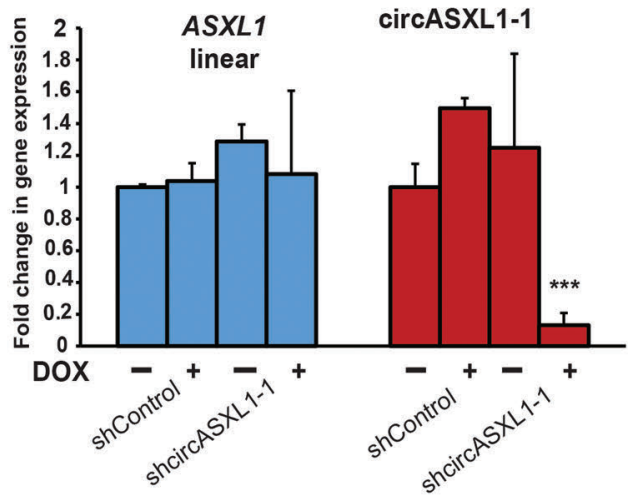

B

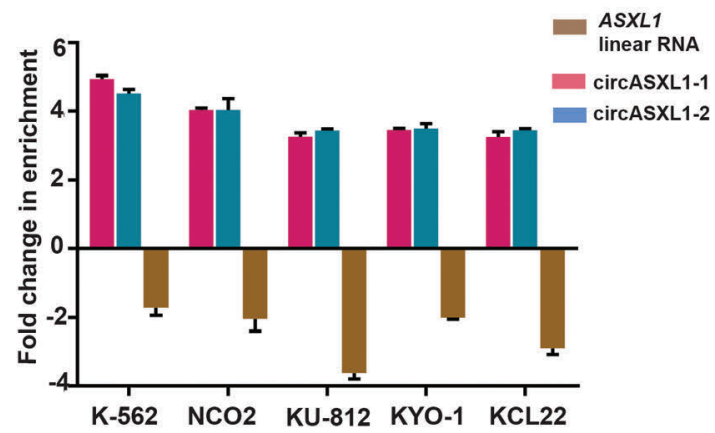

D

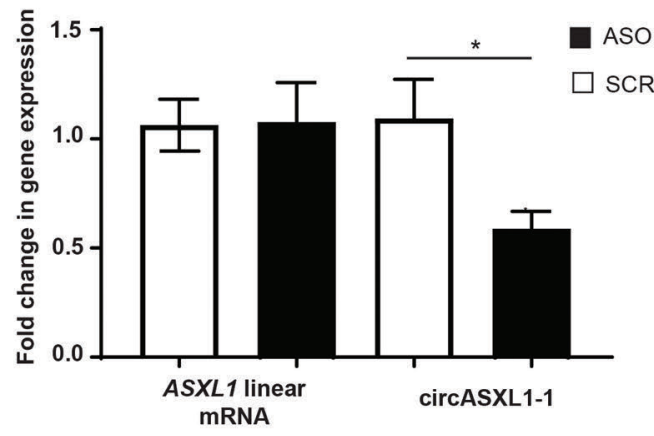

G

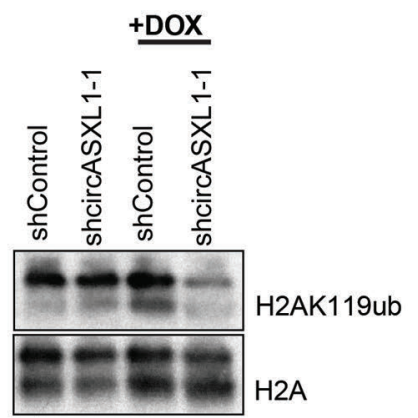

Figure 1. ASXL1 circRNA detected in acute myeloid leukemia and chronic myeloid leukemia cell lines. (A) Expression of ASXL1 linear mRNA, circASXL1-1 and -2 determined using divergent primers in the THP-1 cell line in control and RNAse R-treated samples. (B) Quantitative polymerase chain reaction (PCR) showing enrichment of circASXL1-1 and -2 and degradation of linear ASXL1 mRNA in various chronic myeloid leukemia cell lines. (C) Schematic showing the design of antisense oligonucleotide (ASO) against the backsplice junction of circASXL1-1. (D) Quantitative PCR showing depletion of circASXL1-1 using ASO as compared to scrambled control (SCR). Due to the specific design of the ASO targeted to the backsplice junction of circASXL1-1, no change is observed in corresponding linear mRNA in THP-1 cells. (E) Western blot showing a decrease in H2AK119ub after depletion of circASXL1-1 in THP-1 cells. (F) HEK293 cells with stable depletion of circASXL1-1 after induction with $1 \mu \mathrm{g} / \mu \mathrm{L}$ of doxycycline for $48 \mathrm{~h}$. The shRNA sequence is identical to the ASO design and therefore specific depletion of circASXL1-1 is observed and no change in linear ASXL1 mRNA. (G) H2A immunoprecipitation from HEK293 cells with stable depletion of circASXL1-1 shows a decrease in H2AK119 ubiquitination as compared to shControl cells. Data are represented as mean \pm standard deviation from at least three independent experiments. Statistical analysis was carried out using analysis of variance with a post-hoc Tukey test. $* P<0.05, * * * P<0.001$. 
A

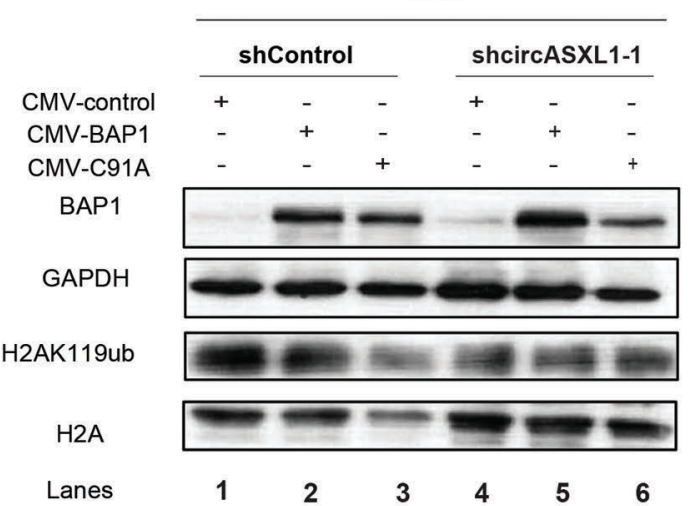

+DoX

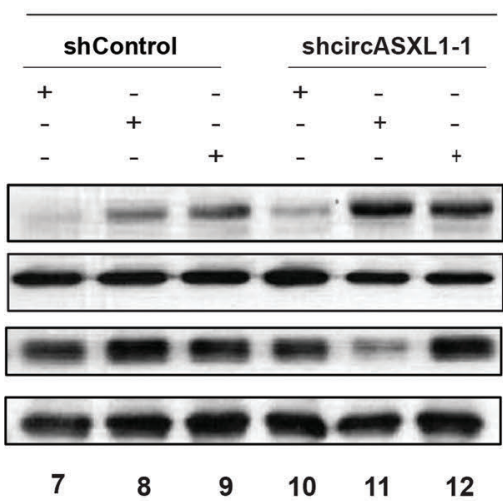

B
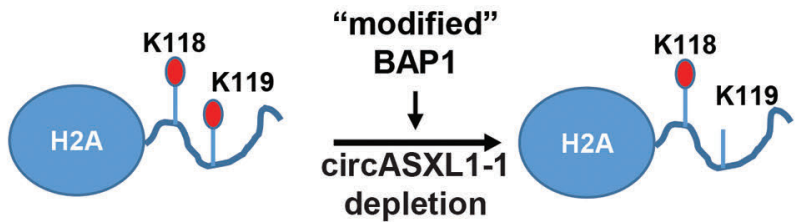

C
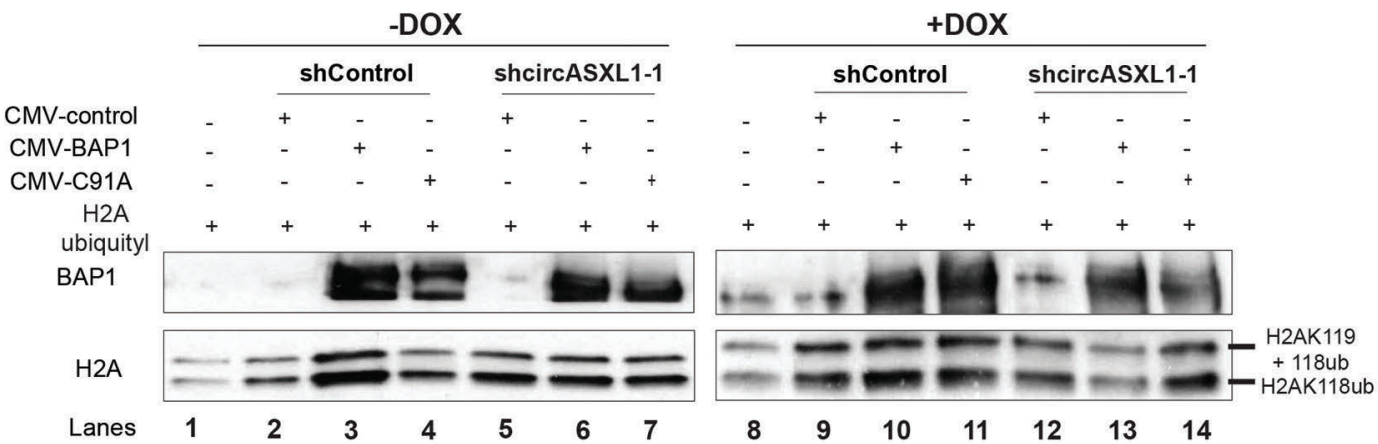

D

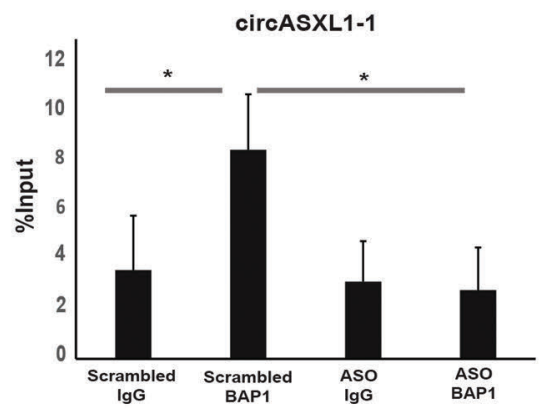

E

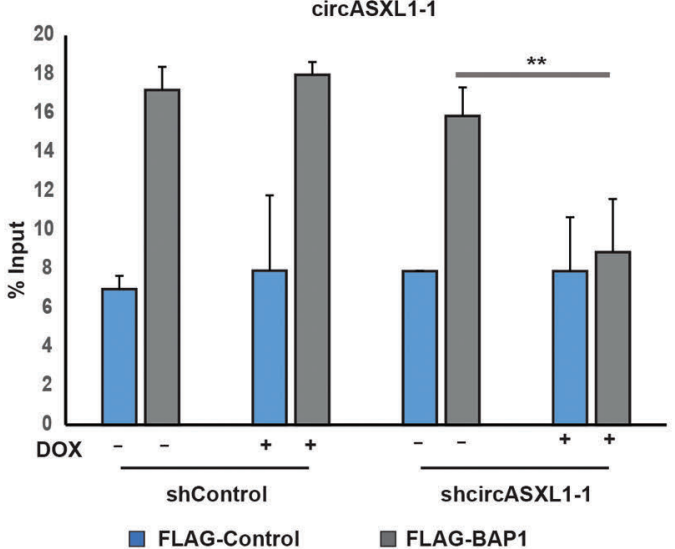

Figure 2. circASXL1-1 regulates BAP1 activity. (A) Western blot showing expression of H2AK119ub in HEK293 cells with stable depletion of circASXL1-1 after overexpression of either cytomegalovirus (CMV)-control or CMV-BAP1 or CMV-C91A constructs. C91A is a catalytic site mutant of BAP1. ${ }^{15}$ (B) Schematic summarizing the experimental design to demonstrate BAP1-mediated deubiquitinase (DUB) activity in the presence or absence of circASXL1-1. The red dots represent the ubiquityl moiety. (C) DUB assay performed by immunoprecipitation of either BAP1 or C91A mutant from HEK293 shControl and shcircASXL1-1 cells with (right panel) or without (left panel) induction with doxycycline. BAP1 purified from cells expressing the indicated shRNA was incubated with recombinant H2AK ubiquityl followed by western blot and probing with total H2A antibody. (D) Quantitative polymerase chain reaction (PCR) showing enrichment of circASXL1-1 after RNA immunoprecipitation using antibody targeting endogenous BAP1 as compared to IgG. (E) Quantitative PCR showing enrichment of circASXL1-1 in HEK293 stable cell lines after transfection of a FLAG-BAP1 construct as compared to empty vector (EV) (control-FLAG). Statistical analysis was carried out using analysis of variance with a post hoc Tukey test. Data are represented as mean \pm standard devaition from at least three independent experiments. $* P<0.05$, $* * P<0.01$. 
A

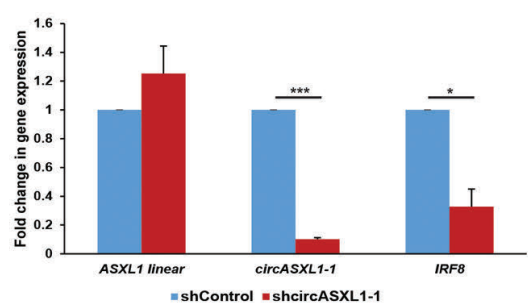

D
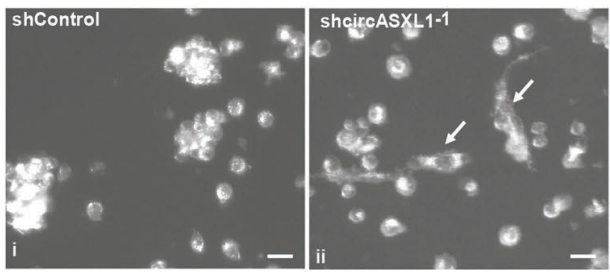

B

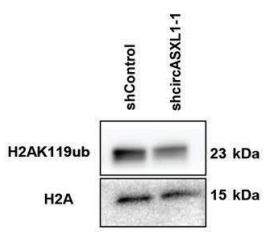

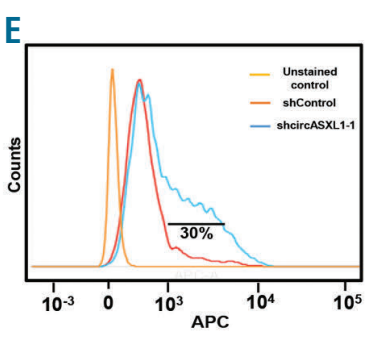

G

Day 0

Isolation of $\mathrm{CD} 34$ cells from healthy individuals
Day 1

Lentiviral transduction

of CD34+ cells with pLKO-GFP-shcontrol and pLKO-GFP-shcircASXL1-1
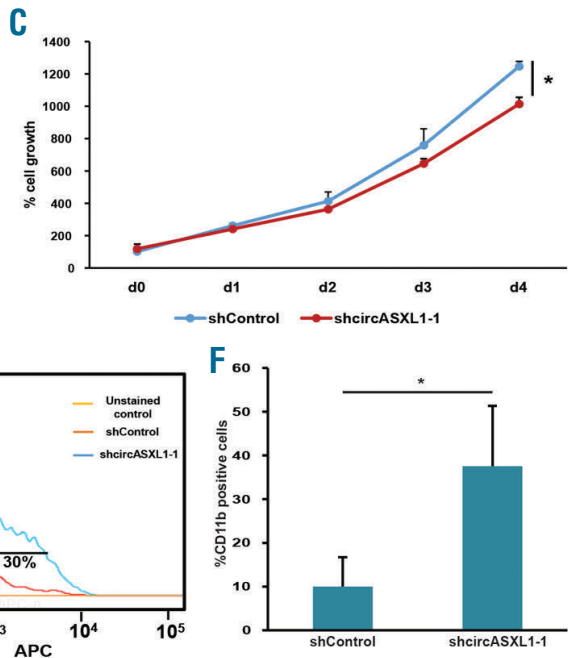

Day 3

Day 16

H

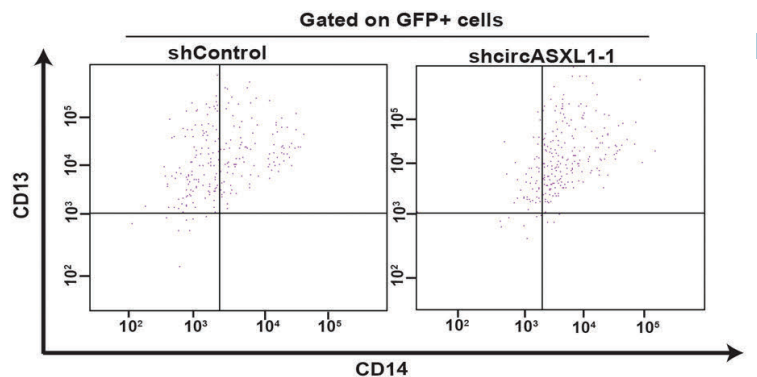

I

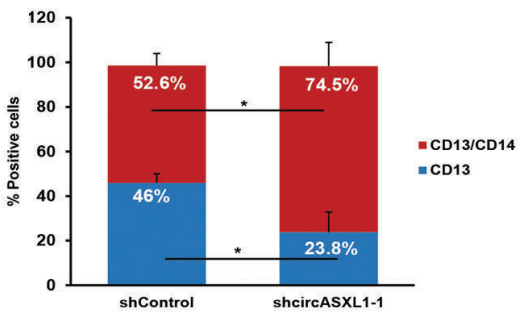

J

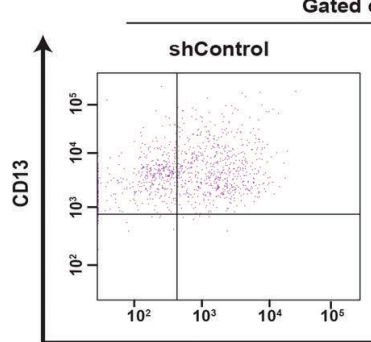

d on GFP+ cells

L

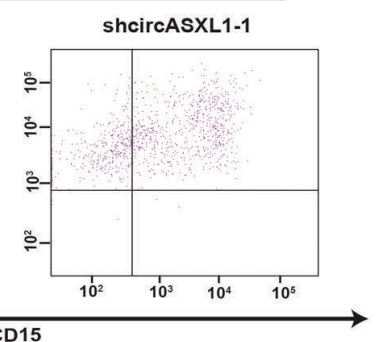

K

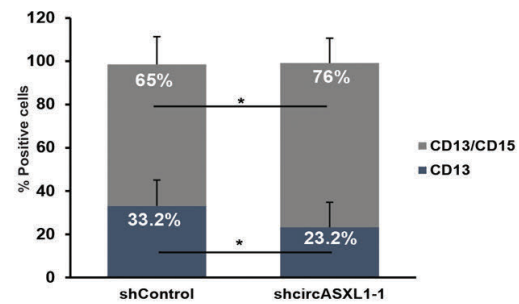

M

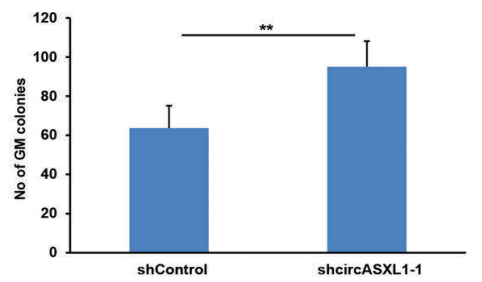

Figure 3. circASXL1-1 affects the growth of leukemic cells and the differentiation of CD34+ progenitors into the myeloid lineage. (A) Quantitative polymerase chain reaction (PCR) analyses showing the expression of genes after depletion of circASXL1-1 as compared to shControl in THP-1 cells. (B) Western blot analyses showing a decrease in H2AK119ub after depletion of circASXL1-1 as compared to control. (C) Cell Titre-Glo assay measuring the growth of shcircASXL1-1 as compared to shControl THP-1 cells over a period of 4 days. (Di-Dii) Phase-contrast images of shControl (i) and shcircASXL1-1 cells (ii). Arrows point to shcircASXL1-1 cells that have undergone differentiation. Scale bar: $50 \mu \mathrm{m}$. (E) Histogram overlay representing the flow cytometric analysis of CD11b intensity across circASXL1-1 and shControl THP-1 cells. (F) Quantification of FACS data showing an increase in CD11b positive shcircASXL1-1 as compared to shControl THP-1 cells. (G) Schematic workflow of the human $\mathrm{CD} 34^{+}$cell transduction and differentiation protocol. (H) Flow cytometry analyses of green fluorescent protein (GFP)-positive mature monocytes using CD13 and CD14 antibodies observed after differentiation of CD34+ bone morrow (BM) progenitors expressing pLKO-GFP shControl shRNA or pLKO-GFP shcircASXL1-1. (I) Quantification of GFP+ monocytes shows an increase in CD13/CD14 double-positive monocytes after circASXL1-1 depletion as compared to shControl. (J) Flow cytometry analyses of GFP-positive mature granulocytes using CD13 and CD15 antibodies observed after differentiation of CD34+ BM progenitors expressing pLKO-GFP shControl or pLKO-GFP shcircASXL1-1. (K) Quantification of GFP+ granulocytes shows an increase in CD13/CD15 double-positive granulocytes after circASXL1-1 depletion as compared to shControl. (L) Phase-contrast images showing granulocyte-macrophage (GM) colonies in shControl (i) and shcircASXL1-1 (ii) CD34 ${ }^{+}$cells. Scale bar: $200 \mathrm{\mu m}$. (M) Quantification of the colonies obtained in (K). Bar graph showing an increase in GM colonies after depletion of circASXL1-1 as compared to shControl. Results are compiled from three independent repeats performed in duplicate. The results were analyzed using Student $t$-test, $* P<0.05$, $* * P<0.01$. 
Recently, the ASXL1 gene locus was shown to undergo alternative splicing to produce circular RNA (circRNA) in addition to the linear protein-coding transcripts.

Covalently closed circRNA function as competing endogenous RNA and microRNA sponges, protein sponges or decoys, and regulators of cell proliferation, splicing and parental gene expression. ${ }^{8}$ The majority of circRNA appear to be more often downregulated in tumor tissue compared to normal tissue, ${ }^{9}$ with evidence demonstrating the role of circRNA in different hallmarks of cancer including cancer initiation and progression, induction of angiogenesis, invasion, and metastasis. ${ }^{10}$ Moreover, circRNA are more stable, more abundant, and better conserved than linear RNA. In addition, circRNA can be detected in extracellular vesicles, exosomes and blood plasma thereby highlighting their potential as noninvasive biomarkers. ${ }^{11}$ In a pioneering study performed by Salzman et al., several circRNA were identified in primary human leukocytes and other blood cells as well as in samples from patients with acute lymphoblastic leukemia. ${ }^{12}$ More recently, fusion circRNA arising from chromosomal translocations in leukemia such as $M L L$ $A F 9$ and PML-RARA have been shown to contribute to cellular transformation and tumorigenesis in vitro and in in vivo mouse models. ${ }^{13}$ In the current study, we sought to identify the functions of ASXL1 circRNA, with the idea of developing new therapeutic targets in acute myeloid leukemia.

By deep RNA sequencing, we identified two putative circRNA isoforms from the ASXL1 gene locus in the THP1 leukemic cell line (Online Supplementary Figure S1A, B). These isoforms could be detected by polymerase chain reaction (PCR) using divergent primers on exon 3 in the THP-1 leukemic cell line followed by visualization on an agarose gel. The amplicons were confirmed to be circRNA after RNAse R treatment which degrades ASXL1 linear RNA while enriching the circRNA isoforms (Figure 1A). Similarly, both circASXL1-1 and -2 were also found to be expressed in a number of chronic myeloid leukemia (CML) cell lines by quantitative PCR and enriched after RNAse $\mathrm{R}$ treatment (Figure 1B). These circRNA isoforms were also found to be expressed in a variety of leukemic cell lines and HEK293 cells (Online Supplementary Figure S1C). The expression of circASXL1-1, -2 and linear ASXL1 mRNA was analyzed in samples from healthy controls and patients with acute myeloid leukemia (AML) by digital droplet PCR (ddPCR). However, no significant change in expression was detected in either the circRNA or linear ASXL1 RNA isoforms in the healthy controls as compared to the AML samples analyzed (Online Supplementary Figure S1D). Isolation of nuclear-cytoplasmic fractions followed by ddPCR analysis demonstrated that circASXL1-1 and circASXL1-2 were localized in the cytoplasm, similarly to linear ASXL1 mRNA (Online Supplementary Figure $S 1 E$ ) with a relative abundance ratio of 100:10:1 of linear to circASXL1-1 and circASXL1-2, respectively (Online Supplementary Figure S1F). Given the relatively low abundance of circASXL1-2, further lossand gain-of-function analyses were focused on circASXL1-1.

Specific depletion of circASXL1-1 was achieved using antisense oligonucleotide (ASO) against the circASXL1-1 backsplice junction (Figure $1 \mathrm{C}$ ). As expected, use of ASO did not affect linear $A S X L 1 \mathrm{mRNA}$ levels as compared to scrambled ASO (Figure 1D). To investigate whether circASXL1-1 had functions in regulating the PR-DUB or Polycomb repressive complex 2 (PRC2) activity, depletion of circASXL1-1 was performed using ASO, followed by western blotting to check H2AK119 ubiquitination and $\mathrm{H} 3 \mathrm{~K} 27 \mathrm{me} 3$ levels. Compared to scrambled treated cells, depletion of circASXL1-1 led to a decrease in H2AK119 ubiquitination (Figure 1E). On the other hand, H3K27me3 did not change significantly as compared to control (Online Supplementary Figure S2A), suggesting that circASXL1-1 affects BAP1 activity but not PRC2 activity in THP-1 leukemic cells. To rule out any off-target activity of ASO and for future mechanistic studies, stable HEK293 cell lines with doxycycline-inducible depletion of circASXL1-1 were generated. Upon induction of short hairpin (sh)RNA expression with doxycycline for $48 \mathrm{~h}$, a significant depletion of circASXL1-1 was observed in shcircASXL1-1 + doxycycline cells as compared to shControl + doxcycline cells with no change in linear ASXL1 mRNA (Figure 1F). ASXL1 protein level also did not change upon depletion of circASXL1-1 with doxycycline (Online Supplementary Figure S2B). Total H2A was immunoprecipitated from these cells and an analysis of the H2AK119ub mark by western blotting indicated a decrease in H2AK119ub after doxycycline-induced stable depletion of circASXL1-1 in HEK293 cells as compared to shControl cells (Figure 1G). These data suggest that circASXL1-1 regulates BAP1-mediated deubiquitinase activity.

To elucidate the mechanism of circASXL1-1-induced modulation of BAP1 activity, either wild-type BAP1, a catalytically inactive BAP1 mutant (C91A) or empty vector constructs (CMV-control) were transfected into doxycycline-inducible shcircASXL1-1 HEK293 cells followed by western blotting to determine H2AK119ub levels. In the absence of doxycycline, H2AK119ub levels remained unchanged both in the shControl and the shcircASXL1-1 cells across all groups (Figure 2A, lanes 1-6). However, when circASXL1-1 was depleted after doxycycline induction for $48 \mathrm{~h}$, a reduction in global H2AK119ub levels was observed in shcircASXL1-1 cells overexpressing BAP1 as compared to shControl cells (Figure 2A, lanes $7-$ 12). Moreover, depletion of H2AK119ub levels was dependent on the catalytic activity of BAP1 since the expression of C91A mutant did not affect H2AK119ub levels in circASXL1-1-depleted cells (Figure 2A, compare lanes 11-12). To further ascertain the modulation of BAP1 activity by circASXL1-1, we carried out an in vitro deubiquitinase assay. For this, FLAG-BAP1 was purified from the lysates prepared from doxycycline-induced shControl or shcircASXL1-1-expressing cells followed by incubation with recombinant $\mathrm{H} 2 \mathrm{~A}$ ubiquityl mononucleosomes (H2AK118 and H2AK119) (Figure 2B). In the absence of doxycycline when the shRNA expression was switched off, there was no appreciable difference in the H2AK119ub levels between shControl and shcircASXL11 groups (Figure 2C, lanes 1-7). However, BAP1 immunoprecipitated from circASXL1-1-depleted HEK293 cells showed more activity than that from shControl cells as observed from a decrease in H2AK119ub when probed with total H2A antibody (Figure 2C, compare lanes 10 and 13) and this was specific to BAP1 catalytic activity as C91A mutant did not show any activity when compared to control lanes (Figure 2C, compare lanes 11 and 14), suggesting that circASXL1-1 regulates BAP1-mediated deubiquitinase activity.

Since circASXL1-1 regulates BAP1 activity, we hypothesized that circASXL1-1 directly binds to BAP1 and inhibits its function. To test this hypothesis, we performed RNA immunoprecipitation of endogenous BAP1 from HEK293 cells expressing either scrambled antisense oligo or an oligo designed against circASXL1-1 backsplice junction (ASO), followed by RNA isolation to identify the RNA binding to BAP1. circASXL1-1 was found to be 
enriched in the BAP1 fraction as compared to the IgG control (Figure 2D). Moreover, ASO-mediated depletion of circASXL1-1 abolished the enrichment with levels of circASXL1-1 comparable to the IgG fraction. Furthermore, HEK293 cells expressing either shcircASXL1-1 or shControl were transfected with control-FLAG (EV) or FLAG-BAP1 followed by immunoprecipitation using FLAG beads. circASXL1-1 was found to be enriched in shControl groups (both with and without doxycycline) and shcircASXL1-1 cells without doxycycline as compared to EV. Moreover, the level of circASXL1-1 was comparable to that in the EV group after circASXL1-1 was depleted using doxycycline treatment for $48 \mathrm{~h}$, thereby showing specificity for the assay. As an additional control for the experiment, depletion of BAP1 using short interfering (si)RNA was performed in HEK293 cells (Online Supplementary Figure S3A). An enrichment of circASXL1-1 was observed in the cells treated with control siRNA using an antibody specific to BAP1 as compared to IgG control. However, loss of BAP1 expression abolished circASXL1-1 enrichment with circASXL1-1 levels being comparable to those in the IgG sample (Online Supplementary Figure S3B). Long non-coding FOXO1 is known to bind to BAP1 and was used as a positive control ${ }^{14}$ (Online Supplementary Figure $3 C, D$ ). Taken together, these results suggest that circASXL1-1 affects BAP1 activity by binding to BAP1, possibly blocking and altering the activity of the PR-DUB complex.

To investigate the role of circASXL1-1 in leukemia, we generated stable cell lines with depletion of circASXL1-1 in THP-1 cells. The efficiency of depletion was confirmed using quantitative PCR (Figure $3 \mathrm{~A}$ ). Moreover, the expression of linear ASXL1 mRNA was unaffected in shcircASXL1-1 cells as compared to shControl (Figure 3A). Consistent with ASO-mediated depletion of circASXL1-1 in THP-1 cells (Figure 1E), western blot analysis indicated that there was a global reduction of histone H2AK119 ubiquitination in shcircASXL1-1 cells as compared to shControl (Figure 3B). Cell growth assays performed over a period of 4 days demonstrated that shcircASXL1-1 cells showed slower growth than shControl cells (Figure 3C). On examining the morphology of the shcircASXL1-1 cells in culture we observed that shcircASXL1-1 cells tended to stick to the Petri dishes and displayed signs of differentiation (Figure 3D panel ii, arrows point to adherent differentiated cells). However, these were not observed in shControl cells which displayed the morphology of cells in suspension growing in clumps (Figure $3 \mathrm{D}$ panel i). To investigate whether shcircASXL1-1 cells were indeed undergoing spontaneous differentiation, we performed fluorescence activated cell sorting (FACS) using CD11b as a marker for measuring differentiation of THP-1 monocytes into macrophages. Our results showed that about $30 \%$ of shcircASXL1-1 cells stained positive for CD11b in the absence of any differentiating agent, indicating that depletion of circASXL1-1 led to spontaneous differentiation of THP-1 cells (Figure 3E, 3F). In addition, we also observed downregulation of the myeloid-specific transcription factor IRF8 in shcircASXL1-1 cells as compared to the levels in shControl (Figure 3A). These data suggest that circASXL1-1 could affect the differentiation of THP1 monocytes by regulating the expression of key genes involved in macrophage differentiation; however further experiments are needed to delineate the transcriptional program and the possible mechanism of action.

Since the PR-DUB complex has been shown to regulate hematopoietic stem cell differentiation, we sought to investigate whether modulation of circASXL1-1 levels affected this differentiation. To this purpose, we depleted circASXL1-1 using pLKO-GFP lentiviruses in CD34 hematopoietic progenitors isolated from the bone marrow of healthy individuals. $\mathrm{CD} 4^{+}$hematopoietic stem cells were allowed to differentiate into granulocytes and monocytes for 14 days followed by FACS analysis to check for cell surface markers on the differentiated cells (Figure 3G). Depletion of circASXL1-1 led to an increase in both CD13/CD14 double-positive mature monocytes (Figure 3H, I) and CD13/CD15 double-positive granulocytes (Figure 3J, K) as compared to shControl cells suggesting that circASXL1-1 influences the differentiation of hematopoietic stem cells towards the myeloid lineage. Colony-forming assays showed an increase in the number of colonies (Figure 3M) without a significant difference in the morphology of the granulocytic-macrophage colonies obtained from $\mathrm{CD} 34^{+}$transduced with shControl virus as compared to shcircASXL1-1 (Figure 3Li-ii).

In conclusion, this study provides a new mechanism for regulation of H2AK119ub levels: via interaction of circASXL1-1 and BAP1. Our results indicate that modulation of circASXL1-1 expression affects BAP1 activity but does not influence PRC2 function. Furthermore, circASXL1-1 binds to BAP1 to regulate its catalytic activity. Our data demonstrate that circASXL1-1 influences the myeloid differentiation of human $\mathrm{CD} 34^{+}$progenitors although the mechanisms underlying the effect of circASXL1-1 on hematopoietic stem cell function and differentiation warrant further investigation. Interestingly, the circASXL1-1 sequence includes an initiation codon along with a Kozak sequence, giving rise to the possibility of circASXL1-1 translating into a peptide. "circASXL11 peptide" could potentially serve as a decoy and compete with ASXL1 protein in binding to BAP1 thus regulating the function of the PR-DUB complex. Regulating BAP1 activity via circASXL1-1 or "circASXL1-1 peptide" could be a promising therapeutic option especially in myeloid malignancies with ASXL1 mutations.

Shweta Pradip Jadhav, ${ }^{1}$ Nishi Kumari, ${ }^{1,2}$ Larry $\mathrm{Ng}^{1,2}$ Pei Fang Tan, ${ }^{3}$ Nicole Shu Ling Yeo-Teh, ${ }^{1,4}$ Yufen Goh, Wee Nih Fam, ${ }^{1}$ Jia Qi Tng, Johann Shane Tian,, Bryan T.H. Koh, ${ }^{5}$ Chan Ming Chun, ${ }^{5}$ Wilson Wang, ${ }^{6}$ Wee Joo Chng, 1,7 Melissa Jane Fullwood, 1,8,9 Ernesto Guccione, ${ }^{9,10}$ Neerja Karnani, ${ }^{3,2}$ Daniel G. Tenen ${ }^{1,11}$ and Sudhakar Jha ${ }^{1,2}$

Cancer Science Institute of Singapore, National University of Singapore, Singapore; 'Department of Biochemistry, Yong Loo Lin School of Medicine, National University of Singapore, Singapore; ${ }^{3}$ Singapore Institute for Clinical Sciences, A*STAR, Singapore; ${ }^{4}$ National University of Singapore Graduate School for Integrative Sciences and Engineering, Singapore; Department of Orthopedic Surgery, National University Health Systems, Singapore; ${ }^{6}$ Department of Orthopedic Surgery, National University of Singapore, Singapore, ${ }^{7}$ Department of Hematology-Oncology, National University Cancer Institute, Singapore; ${ }^{8}$ School of Biological Sciences, Nanyang Technological Institute, Singapore; "Institute of Molecular and Cell Biology (IMCB), A*STAR, Singapore; ${ }^{10}$ Tisch Cancer Institute, Department of Oncological Sciences and Department of Pharmacological Sciences, Icahn School of Medicine at Mount Sinai, New York, NY, USA and "Harvard Stem Cell Institute, Harvard Medical School, Boston, MA, USA

Acknowledgments: we thank the staff of Jha's laboratory for helpful discussion and comments.

Funding: SJ (R-713-006-014-271 and R-713-103-006-135), DGT, WJC, and MJF were supported by grants from the National Research Foundation Singapore and the Singapore Ministry of 
LETTERS TO THE EDITOR

Education under its Research Centers of Excellence initiative to the Cancer Science Institute of Singapore. SJ, DGT, EG, WJC, and MJF were supported by the RNA Biology Center at Cancer Science Institute of Singapore, National University of Singapore, with funding from the Singapore Ministry of Education's AcRE Tier 3 grants (MOE2014T3-1-006). DGT was supported by the Singapore Ministry of Health's National Medical Research Council through a Singapore Translational Research (STaR) Investigator Award and by National Institutes of Health/National Cancer Institute grant R35CA197697.

Correspondence: SUDHAKARJHA - csisjha@nus.edu.sg doi:10.3324/haematol.2019.225961

Information on authorship, contributions, and financial \& other disclosures was provided by the authors and is available with the online version of this article at www. haematologica.org.

References

1. Gelsi-Boyer V, Brecqueville M, Devillier R, Murat A, Mozziconacci MJ, Birnbaum D. Mutations in ASXL1 are associated with poor prognosis across the spectrum of malignant myeloid diseases. J Hematol Oncol. 2012;5:12.

2. Abdel-Wahab O, Adli M, LaFave LM, et al. ASXL1 mutations promote myeloid transformation through loss of PRC2-mediated gene repression. Cancer Cell. 2012;22(2):180-193.

3. Balasubramani A, Larjo A, Bassein JA, et al. Cancer-associated ASXL1 mutations may act as gain-of-function mutations of the ASXL1BAP1 complex. Nat Commune. 2015;6:7307.

4. Asada S, Goyama S, Inoue D, et al. Mutant ASXL1 cooperates with BAP1 to promote myeloid leukaemogenesis. Nat Commun. 2018;9(1):2733.
5. Nagase R, Indue D, Pastor A, et al. Expression of mutant Asxl1 perturbo hematopoiesis and promotes susceptibility to leukemic transformation. J Exp Med. 2018;215(6):1729-1747.

6. Gur Y, Yang H, Chen S, et al. Reduced BAP1 activity prevents ASXL1 truncation-driven myeloid malignancy in vive. Leukemia. 2018;32(8):1834-1837.

7. Kob W, Gonzalez V, Natarajan S, Carter R, Brown PO, Gawad C. Dynamic ASXL1 exon skipping and alternative circular splicing in single human cells. PLo One. 2016;11(10):e0164085.

8. Wilusz JE. A 360 degrees view of circular RNA: from biogenesis to functions. Wiley Interdiscip Rev RNA. 2018;9(4):e1478.

9. Bachmayr-Heyda A, Reiner AT, Auer K, et al. Correlation of circular RNA abundance with proliferation-exemplified with colorectal and ovarian cancer, idiopathic lung fibrosis, and normal human tissues. Sci Rep. 2015;5:8057.

10. Kristensen LS, Hansen TB, Ven MT, Kjems J. Circular RNA in cancr: opportunities and challenges in the field. Oncogene. 2018;37(5):555-565.

11. Li X, Yang L, Chen LL. The biogenesis, functions, and challenges of circular RNAs. Mol Cell. 2018;71(3):428-442.

12. Salzman J, Gawd C, Wang PL, Lacayo N, Brown PO. Circular RNA are the predominant transcript isoform from hundreds of human genes in diverse cell types. PLoS One. 2012;7(2):e30733.

13. Guarnerio J, Bezzi M, Jeong JC, et al. Oncogenic role of fusioncircRNAs derived from cancer-associated chromosomal translocatons. Cell. 2016;165(2):289-302.

14. Xi J, Fen J, Li Q, Li X, Zing S. The long non-coding RNA IncFOXO1 suppresses growth of human breast cancer cells through association with BAP1. Int J Oncol. 2017;50(5):1663-1670.

15. Ventii KH, Devi NS, Friedrich KL, et al. BRCA1-associated protein-1 is a tumor suppressor that requires deubiquitinating activity and nuclear localization. Cancer Res. 2008;68(17):6953-6962.

haematological 2020; 105:e348 